\title{
LA VÉRITÉ DU DRAME
}

Lire le texte dramatique (Dom Juan)

Romain Bionda

Le Seuil | « Poétique »

2017/1 n $181 \mid$ pages 67 à 82

ISSN 1245-1274

ISBN 9782021340624

Article disponible en ligne à l'adresse :

http://www.cairn.info/revue-poetique-2017-1-page-67.htm

\section{Pour citer cet article :}

Romain Bionda, « La vérité du drame. Lire le texte dramatique (Dom Juan) », Poétique 2017/1 (n¹81), p. 67-82.

Distribution électronique Cairn.info pour Le Seuil.

(C) Le Seuil. Tous droits réservés pour tous pays.

La reproduction ou représentation de cet article, notamment par photocopie, n'est autorisée que dans les limites des conditions générales d'utilisation du site ou, le cas échéant, des conditions générales de la licence souscrite par votre établissement. Toute autre reproduction ou représentation, en tout ou partie, sous quelque forme et de quelque manière que ce soit, est interdite sauf accord préalable et écrit de l'éditeur, en dehors des cas prévus par la législation en vigueur en France. Il est précisé que son stockage dans une base de données est également interdit.

Résumé :

Chacun de leur côté, un historien reconnu (Fernand Hallyn) et un célèbre metteur en scène (Jean-Pierre Vincent) ont récemment suggéré qu'il est possible que Dom Juan ne meure pas à la fin de la pièce de Molière - alors que le texte dit explicitement le contraire. Cette hypothèse procède-t-elle d'une infidélité de lecture ? Produit-elle un contresens ? Elle se fonde en tout cas sur une manière de lire les textes dramatiques qui, en France, est encouragée. Après avoir montré la différence entre le fait de lire un texte dramatique et le fait de le lire comme un texte de théâtre — texte qu'on peut alors lire au passé, au futur antérieur ou au futur simple-, cet article prête attention aux interférences que ces manières de lire peuvent produire entre elles dans le présent de la lecture. Il explique ainsi pourquoi il n'est pas simple de s'accorder sur un fait en apparence aussi facile à établir que la mort d'un personnage dans une pièce de théâtre. Par là, il invite à considérer les incidences que cette spécificité de la lecture des textes dramatiques (non naturelle, mais tributaire d'une histoire éditoriale, théâtrale, critique, théorique...) peut avoir sur l'exercice de l'analyse de texte. 


\section{Romain Bionda La vérité du drame}

\section{Lire le texte dramatique (Dom Juan)}

Dans un article relevant de l'histoire littéraire, Fernand Hallyn proposait d'interpréter la mort de Dom Juan, dans la pièce de Molière, «sans y chercher nécessairement l'expression d'une conviction religieuse ${ }^{1} »$ : cette mort peut être comprise "comme la condamnation d'une forme d"athéisme" par une autre, [...] exprimée de manière symbolique et dramatique par l'intervention de la statue", c'est-à-dire comme la condamnation de «l'anarchisme de Dom Juan ${ }^{2}$ " par un athéisme comparable à celui de François de La Mothe Le Vayer, libertin «érudit» dont Molière était proche. Comment dès lors comprendre la contre-proposition suivante, pour le moins surprenante, qui forme la clausule de l'article?

Ou bien... La fin de la pièce de Molière est extrêmement abrupte. Le spectateur ne peut-il pas se poser des questions? Dom Juan avait fait part, un peu plus tôt, de sa décision de disparaitre, d'adopter un mode de vie hypocrite, parce que trop de dangers le menaçaient $(\mathrm{V}, 2)$. Qui peut prétendre dès lors que la pièce se termine réellement sur une punition surnaturelle ou symbolique? N'est-il pas tout aussi bien possible que toute la série des apparitions ait été mise en scène par Dom Juan lui-même, pour lui permettre de disparaitre littéralement et de recommencer, alors que tout le monde le croit mort, une nouvelle vie, toute dévote en apparence?

Dans ce cas, Molière aurait pu imaginer une suite. Mais elle existait déjà plus ou moins: Le Tartuffe. D’autres étaient possibles. Max Frisch en écrira une avec son Don Juan oder die Liebe zur Geometrie (1952) ${ }^{3}$.

Que Le Tartuffe puisse être lu comme la suite de Dom Juan, soit. Mais quid de l'idée selon laquelle le personnage de Dom Juan aurait mis en scène son exécution et simulé sa propre mort? En fait, il paraît difficile, en 1665, que Dom Juan puisse échapper à la mort: le sujet l'impose traditionnellement et aucun témoignage ne

1. Fernand Hallyn, «La dernière ruse de Dom Juan?», p. 203, dans Ulrich Döring, Antiopy Lyroudias, Rainer Zaiser (dir.), Ouverture et dialogue, Tübingen, Gunter Narr, 1988, p. 199-206. Repris dans Pierre Ronzeaud (éd.), Molière. Dom Juan, Paris, Klincksieck, 1993, p. 107-112.

2. Ibid., p. 204.

3. Id. 
suggère que la pièce de Molière ait pu se démarquer sur ce point ${ }^{1}$. Par ailleurs, rien dans le texte ne donne explicitement prise à l'hypothèse de cette mystification dernière. Outre les nombreux effets d'annonce qui la préparent, la mort de Dom Juan est - dans cet ordre - signalée à la dernière scène de l'acte $V$ par Dom Juan lui-même ( $\mathrm{O}$ Ciel que sens-je? un feu invisible me brûle, je n'en puis plus et tout mon corps devient... ${ }^{2}$ ), reconnue telle par son valet Sganarelle ("voilà par sa mort un chacun satisfait») et confirmée par la didascalie finale ("Le tonnerre tombe avec un grand bruit et de grands éclairs sur Dom Juan; la terre s'ouvre et l'abîme; et il sort de grands feux de l'endroit où il est tombé»). Qui peut prétendre dès lors que Dom Juan ne meurt pas ${ }^{3}$ ?

L'affaire trouve un prolongement dans une actualité récente. En 2012 à la ComédieFrançaise, le metteur en scène Jean-Pierre Vincent fait se relever Dom Juan à la toute fin de la pièce. La critique théâtrale du Figaro le lui reproche, arguant qu'en laissant "le grand seigneur scélérat [...] reprend[re] tranquillement sa route», «le sens de l'œuvre» est «transform[é]». Or:

Quand on aime Molière, quand on admire son courage intellectuel, politique, quand on respecte sa pensée, ses convictions, on est blessé par le trait de plume qui fait disparaitre la conclusion qu'il a souhaite $[s i c]^{4}$.

Bien qu'elles soient d'une tout autre nature et qu'elles jouissent a priori, notamment sur le plan de la liberté interprétative, d'un autre statut, peut-on dire que les propositions de Hallyn et de Jean-Pierre Vincent procèdent d'une analogue infidélité de lecture ou qu'elles produisent un contresens?

Gardons-nous toutefois de déclarer trop expéditivement l'hypothèse de Hallyn infondée, et demandons-nous plutôt en vertu de quoi le lecteur d'un texte dramatique (en l'occurrence de Dom Juan) serait encouragé, voire autorisé à douter de ce quil lit. C'est en nous demandant (laissons dans un premier temps de côté la mise en scène de Jean-Pierre Vincent) sur quoi exactement porte l'hypothèse de Hallyn (le monde de la fiction, le texte, ou la mise en scène?) et quelles sont ses implications en tant que geste critique que nous découvrirons progressivement pourquoi il n'est pas facile de s'accorder sur un fait aussi simple que la mort d'un personnage... dans une pièce de théatre.

1. Un contemporain anonyme rapporte que le roi lui-même aurait livré cette interprétation de la fin de la pièce: «[...] je ne crois pas avoir beaucoup de choses à y répondre, quand j’aurai dit, après le plus grand monarque du monde, qu'il [Don Juan] n'est pas récompensé [de ses vices]. " (Lettre sur les Observations d'une comédie du Sieur Molière, intitulée "Le Festin de Pierre", p. 1233, dans Molière, Euvres complètes II, Georges Forestier, Claude Bourqui [éd.], Paris, Gallimard, 2010, p. 1229-1241).

2. Molière, Le Festin de Pierre [Don Juan], in CEuvres complètes II, op. cit., p. 845-902.

3. Signalons que la didascalie finale est absente de l'édition d'Amsterdam (1683), sans doute plus fidèle au texte joué en 1665 que la première édition de 1682, et que les deux impressions sont posthumes. Sur les différentes versions du texte, voir la notice des Euvres complètes II, op. cit., p. 1619-1648. Hallyn ne précise pas quelle édition il lit.

4. Armelle Héliot, "Dom Juan, de la plus grande fidélité à la trahison finale...» (23 septembre 2012), dans Le Grand Théâtre du monde, blog hébergé sur le site du Figaro. En ligne: http://blog.lefigaro.fr/ theatre/2012/09/dom-juan-de-la-plus-grande-fid.html. Consulté le 24 février 2017. 


\section{La vérité du récit}

La question de la "valeur de vérité» des énoncés d'un texte dramatique a été posée au moins une fois en ces termes dans un essai lui-même surprenant de François Rastier, intitulé «L'ambiguïté du récit: la double lecture de Dom Juan de Molière». On prendra le temps de le suivre.

F. Rastier y plaide pour l'ouverture d'une «théorie de la lecture» qui «permet[te] d'étendre aux textes littéraires la technique de description des récits élaborée par les folkloristes", aux rangs desquels Algirdas Julien Greimas : "Rien ne sert d'hypostasier l'ambiguiité des textes dits littéraires: il faut montrer comment elle est un effet de certaines structures discursives ${ }^{1}$.» Pour ce faire, le sémioticien tente d'isoler la "vérité du récit ${ }^{2}$ ", aussi désignée "vérité-du-récit ${ }^{3}$ " et "vérité-de-récit ${ }^{4}$ ", des signes et des énoncés dont la "valeur de vérité» reste «indéterminée ${ }^{5}$ ». Il explique que la

valeur de vérité d'un énoncé est défini[e] par la cohérence de ses investissements de contenus avec des contenus de référence (ceux des propos du narrateur principal, par exemple) et non par référence à une quelconque réalité extralinguistique [...]. En relèvent tous les contenus qui entrent dans la composition de toute isotopie de lecture cohérence $[s i c]$ construite à partir du texte. [...] Si aucune isotopie cohérente ne peut être construite [...], la valeur de vérité de tous les énoncés est indécidable ${ }^{6}$.

En d'autres termes, sont exclus de la "vérité du récit» tous les contenus considérés comme "contestable $[s]]^{7}$ » en regard "des contenus de référence». F. Rastier écrit au terme de son étude: "Dans la mesure où la lecture totalisante ici produite est scientifique, elle permet de dévoiler comme idéologiques les lectures partielles ${ }^{8}$. " La mort de Dom Juan relève-t-elle de la "vérité scientifique du récit» ainsi comprise? La lecture de Hallyn et la mise en scène de J.-P. Vincent seraient-elles dès lors à dénoncer comme idéologiques?

Comment F. Rastier procède-t-il ? On s'étonnera d'abord que la "vérité du récit» est théorisée à partir d'un texte dramatique. F. Rastier explique d'ailleurs lui-même

1. François Rastier, "L'ambiguïté du récit: la double lecture de Dom Juan de Molière», p. 161, Essais de sémiotique discursive, Tours, Maison Mame, 1973, p. 91-161. En ligne: http://www.revue-texto.net/ Parutions/Essais-de-semiotique/pages089-161.pdf. Consulté le 24 février 2017. Article paru initialement dans Semiotica III, 4, La Haye, Mouton, 1971, p. 289-342, sous le titre "Les niveaux d'ambiguïté des structures narratives".
2. Ibid., p. 95.
3. Ibid., p. 150.
4. Ibid., p. 160.
5. Ibid., p. 94.
6. Id. Lauteur souligne.
7. Ibid., p. 113.
8. Ibid., p. 161. 
que la lecture du «récit» d'un «texte de genre dialogique» présente des difficultés spécifiques:

Par rapport aux propos des acteurs du récit, les propos d'un narrateur unique non présent dans le récit peuvent avoir une fonction métalinguistique: ils présentent des énoncés indépendants des acteurs et qui permettent d'identifier comme des mensonges les énoncés non cohérents avec eux. En revanche, dans un texte de genre dialogique, la valeur de vérité d'un énoncé peut être indéterminée, si elle n'est pas précisée par les indications scéniques ou les propos d'un récitant extérieur au récit ${ }^{1}$.

En effet,

dans le texte de genre dialogique, et notamment ceux qui sont destinés au théâtre, seules les indications scéniques et, éventuellement, les paroles d'un récitant, assurent la fonction de narration sans que le narrateur soit présent comme acteur dans le récit; cela mis à part, elle est assurée par les acteurs du récit lui-même: les locuteurs coïncident avec les narrateurs, les acteurs de l'énoncé avec des acteurs de l'énonciation. Or, sil y a plusieurs narrateurs, on peut avoir plusieurs versions du récit. On ne sait plus alors s'il est possible de constituer un modèle qualificatif unique qui rende compte de toutes les versions du récit, et d'identifier un récit de base à partir duquel on pourrait produire, par transformation, toutes les versions manifestées ${ }^{2}$.

En l'absence d'un narrateur unique qui «assure un encadrement homogène des énoncés ${ }^{3}$ ", que faire? Le sémioticien "propose d'établir si le contenu litigieux est reconnu par des narrateurs actants opposés, c'est-à-dire s'il est présent dans leurs propos sur un mode assertorique et non déceptif ${ }^{4} »$. Exemple:

le mal fait par Don Juan [chez Tirso de Molina] est [...] Reconnu par tous, et l'on peut dire alors qu'il appartient à la vérité du récit. De la même façon, dans le Dom Juan de Molière, Sganarelle et Dom Juan, qui sont, comme on le montrera plus loin, des actants opposés, parlent à l'acte II, scène 2, d'un naufrage qu'ils ont subi. Le naufrage appartient à la vérité du récit. Mais Sganarelle voit dans leur salut un signe de la pitié céleste, ce que Dom Juan ne fait pas. La valeur de vérité, dans le récit, de ce signe céleste est donc indéterminée 5 .

Quelles sont les conclusions de F. Rastier? Deux résultats de sa longue étude nous intéressent en priorité. D'abord, l'hypocrisie jouit d'un statut particulier, dans la mesure où

1. Ibid., p. 94.

2. Ibid., p. 93-94. Je souligne.

3. Ibid., p. 93.

4. Ibid., p. 94.

5. Ibid., p. 95. Les petites majuscules sont de F. Rastier. 
c'est le seul contenu [...] reconnu par deux locuteurs actants opposés (Dom Juan et Sganarelle, V, 2); de plus il est à deux reprises attribué à Dom Juan par des indications scéniques $(\mathrm{V}, 1 ; \mathrm{V}, 3)$; c'est donc le seul contenu des deux systèmes sémiologiques qui appartienne, indéniablement d'ailleurs, à la vérité-de-récit ${ }^{1}$.

Ensuite la valeur de vérité du Ciel est indéterminée, dans la mesure où

la Statue et [le] Spectre [...] font partie de l'irrationnel: il s'agit de "quelque chose» que Dom Juan «ne comprend pas» $(\mathrm{V}, 2)$. Le Spectre n'est pas identifié: "Spectre, fantôme ou diable, je veux voir ce que c'est» $(\mathrm{V}, 5)$.

Les fonctions de ces deux acteurs appartiennent seules à la vérité-du-récit, non leurs motivations, car les contenus qu'ils assument ne sont pas reconnus par Dom Juan ${ }^{2}$.

Observons que pour F. Rastier il semble bien que «la foudre brûle Dom Juan» et que «Dom Juan n'est plus ${ }^{3}$ " pour de vrai: ce seraient des faits (ou des "fonctions» du récit appartenant à sa "vérité») - mais dont le "Ciel» ne serait pas l'origine assurée (ou la «motivation»).

Il faut risquer à ce stade trois remarques. 1) Malgré l'apparent accord entre F. Rastier et Hallyn sur ce point, il n'est pas sûr que la valeur de vérité du Ciel dans le texte soit réellement indéterminée. 2) Il n'est pas davantage certain que la vérité du récit tel qu'il est écrit soit la seule "vérité" à prendre en compte, car Dom Juan est représenté non seulement textuellement (il est écrit), mais encore scéniquement (il est joué). 3) C'est peut-être la prise en compte d'une représentation théâtrale qui encourage Hallyn à aller dans sa lecture du texte plus loin que F. Rastier en supposant que la mort de Dom Juan puisse être feinte.

\section{La vérité du texte dramatique}

Sauf à croire que Dom Juan feigne la mort avec la complicité du Ciel (hypothèse conspirationniste), il faut que la Statue et le Spectre ne soient pas (ou pas assurément) des manifestations dudit Ciel pour qu'il soit envisageable que Dom Juan survive à ce qu'il convient sinon de qualifier de châtiment divin. Si l'on suit F. Rastier dans son analyse du texte, la condition est remplie: dès lors que la valeur de vérité du Ciel se révèle indéterminée, il serait "bien possible», pourquoi pas?, " que toute la

1. Ibid., p. 160. F. Rastier résume alors: «Le texte de la pièce manifeste donc deux structures narratives différentes, qui, interprétées par deux systèmes sémiologiques opposés, articulent deux messages de sens inverse, qui ne coïncident que sur un point, la punition de l'hypocrisie, seul contenu qualificatif non ambigu.»

2. Ibid., p. 150. En note: «Notons que les fonctions de la Statue sont d'abord non reconnues (IV, 1), puis reconnues au moment de la confirmation du contrat (IV, 7).»

3. Ibid., p. 111. 
série des apparitions ait été mise en scène par Dom Juan lui-même» (Hallyn). Mais est-on bien sûr que le Ciel n'appartient pas à la vérité du récit?

Sganarelle, d'abord, prévoit la condamnation de son maître - «[...] il faut que le courroux du Ciel l'accable quelque jour" $(\mathrm{I}, 1)$-, puis tente de l'en avertir à la suite d'un vif échange avec lui : "croyez ce que vous voudrez: il m'importe bien que vous soyez damné» (III, 1). Il diagnostique immédiatement une origine céleste aux événements lorsqu'ils surviennent: «je ne doute point que le Ciel scandalisé de votre vie n'ait produit ce miracle pour vous convaincre» (IV, 1). La Statue, ensuite, corrobore cette interprétation en prétendant être "conduit[e] par le Ciel» (IV, 8). Le Spectre enfin déclare être ce devant quoi / qui Dom Juan peut en se repentant "profiter de la miséricorde du Ciel» $(\mathrm{V}, 5)$ dont (commente enfin la Statue) «les grâces $[\ldots]$ que l'on renvoie ouvrent un chemin à la foudre» $(\mathrm{V}, 6)$. Les trois versions $d u$ "récit» de Sganarelle, de la Statue et du Spectre concordent donc.

Qu'en est-il maintenant de la version de Dom Juan? Le libertin semble admettre par deux fois avoir perçu quelque chose: avant de réclamer le souper - «Quoi qu’il en soit, laissons cela, c'est une bagatelle, et nous pouvons avoir été trompés par un faux jour, ou surpris de quelque vapeur qui nous ait troublé la vue» (IV, 1) -, et lorsqu'il reconnaît dans le mouvement de tête de Sganarelle celui de la Statue:

Dom Juan: Qu'est-ce donc, qu’y a-t-il là?

SGANARELLE, baissant la tête: Le... qui est là.

Dom JuAn : Allons voir, et montrons que rien ne [me] saurait ébranler ${ }^{1}$. (IV, 7)

Par ailleurs, il converse bel et bien avec la Statue et avec le Spectre. Faut-il suivre F. Rastier dans sa distinction entre les «fonctions» et les «motivations» de ces deux "acteurs", dont les premières seules appartiendraient à la "vérité du récit", car Dom Juan ne reconnaitrait pas les secondes (la Statue et le Spectre agiraient bien, mais sans que le Ciel en décide assurément)? Dans sa dernière réplique, Dom Juan ne sent-il pas un «feu invisible» qui le "brûle», après s'être exclamé "O Ciel» $(\mathrm{V}, 6)$ ? Est-on certain qu'il n'assigne pas alors à ce feu une origine céleste? Les motivations de la Statue et du Spectre ne pourraient-elles alors pas être reconnues in fine comme divines? Tous les personnages n'admettraient-ils pas (in)directement son existence? Il faut en réalité avouer que la version du "récit» de Dom Juan est pour le moins ambiguë - peut-être joue-t-il la comédie: il est hypocrite - et qu'il est dès lors inenvisageable de nous déterminer sur la question.

Si l'on se fie toutefois aux didascalies d'attribution, le spectre est appelé «Spectre». Peut-il alors être un simple pantin, un reflet ou un automate - Dom Juan donne le choix suivant: "spectre, fantôme ou diable» $(\mathrm{V}, 5)$-, à l'instar de la Vénus des Amants magnifiques? Mais si dans ce dernier cas, précisément, la nature artificielle de la Vénus est explicitement assumée par le texte didascalique - elle est "accompagnée de quatre petits Amours dans une machine» (IV, 2) - et par son utilisateur Anaxarque

1. Les versions de 1682 précisent: «baissant la tête comme a fait la statue». Voir la note dans l'édition citée des Euvres complètes II, p. 1662. 
$(\mathrm{IV}, 3)^{1}$, le texte didascalique des éditions de 1682 de Dom Juan suggère plutôt le contraire, dans la mesure où semblent être attribuées au Spectre des capacités surhumaines: celui-ci «change de figure et représente le Temps avec sa faux à la main» et "s'envole» $(\mathrm{V}, 5)$. La didascalie finale le confirme enfin: la foudre s'abat sur Dom Juan avant qu'un feu souterrain ne l'engouffre. Si, comme l'explique F. Rastier, le texte didascalique assure "une fonction métalinguistique» (ou métatextuelle) dans le texte dramatique, alors il confère une valeur de vérité aux manifestations du surnaturel, dont l'origine paraît céleste à bien des égards : le feu (Dom Juan dit en sentir l'invisible puissance) et la foudre sont les attributs du Ciel. En considérant les éditions de 1682 - ce que, précisément, F. Rastier ne fait pas: il lit l'édition de 1683, dépourvue de la plupart des didascalies ${ }^{2}-$, peut-on encore conclure à l'ambiguïté du récit sur ce point? Le texte didascalique de 1682 arbitre même immédiatement le débat entre le personnage éponyme et son valet au sujet de la Statue et du Spectre: "La Statue baisse la tête» (III, 5). Les énoncés des personnages qui contredisent les énoncés métatextuels ne devraient-ils pas être identifiés comme des «mensonges" par le lecteur - ou ici, plutôt que comme des «ruses", comme des erreurs? Par ailleurs, de toute façon, pourquoi faire crédit à la version du personnage de Dom Juan, que l'animation effective de la Statue relativise bien vite? Ainsi, bien que le libertin ait pu paraître à certains critiques imperméable au doute, remarquons qu'il aurait raison d'hésiter, puisqu'il a tort sur une bonne partie, sinon sur tout ce qui concerne l'au-delà et ses manifestations. Son point de vue est-il dès lors vraiment fiable sur le plan de la "vérité du récit»?

Pourtant, même Georges Couton - qui, à l'exception de l'indication finale, transcrit dans son édition les didascalies de 1682 - est à deux doigts de céder au doute:

Un Dom Juan auquel l'hypocrisie enlève la sympathie à quoi il pouvait prétendre, s'il se fût hardiment révolté; un Sganarelle pourvu de cette foi si enviable qu'est celle du charbonnier; un dénouement qui punit par une mauvaise mort une mauvaise vie et qui envoie aux enfers le pécheur impénitent vainement sommé de se repentir et qui a refusé la grâce; voilà une pièce plus qu'irréprochable, édifiante.

Ne l'est-elle pas trop ${ }^{3}$ ?

1. «Enfin, mon fils, comme nous venons de voir par cette ouverture, le stratagème a réussi, notre Vénus a fait des merveilles; et l'admirable Ingénieur qui s'est employé à cet artifice a si bien disposé tout, a coupé avec tant d'adresse le plancher de cette Grotte, si bien caché ses fils de fer et tous ses ressorts, si bien ajusté ses lumières, et habillé ses Personnages, qu'il y a peu de gens qui n’y eussent été trompés. [...] Il y a longtemps, mon fils, que je prépare cette machine [...]" (Molière, Les Amants magnifiques, p. 985, in Euvres complètes II, op. cit., p. 943-995).

2. F. Rastier, art. cité, p. 98. Malgré ce qu'il en dit à la p. 160, les didascalies qui précisent l'hypocrisie de Dom Juan sont absentes de l'édition de 1683, selon G. Forestier et C. Bourqui (in CEuvres complètes II, op. cit., p. 1664). Pour rappel, F. Rastier utilisait leur présence comme un argument supplémentaire pour reconnaître à l'hypocrisie une valeur de vérité (voir passage déjà cité, p. 160).

3. Georges Couton, «Préface», p. 18-19, dans Molière, Dom Juan, G. Couton (éd.), Paris, Gallimard, 1971, actualisée par Carine Barbafieri (1999), p. 7-24. Le texte de la préface est raccourci par rapport à celui de 1971. 
Pourquoi ne serait-elle pas édifiante ${ }^{1}$ ? Est-ce à dire que le texte didascalique, censé "assurer la fonction de narration", vecteur d'un "contenu de référence», est frappé du sceau du soupçon et, au même titre que les énoncés pris en charge par de simples personnages, indigne de confiance? La mystification orchestrée par Dom Juan s'étendrait-elle jusqu'aux énoncés didascaliques? Le personnage hypocrite serait ainsi capable de tromper non seulement les autres personnages, mais encore les lecteurs du texte (l'auteur serait alors de mèche - à ce stade, il faut tout supposer ${ }^{2}$ ) ? Ou bien le texte didascalique est-il la "dernière ruse» non de Dom Juan, mais des éditeurs de 1682 pour contraindre le potentiel subversif du texte?

On suivra ici une autre piste: faisons l'hypothèse que si les énoncés didascaliques de Dom Juan ne constituent pas, aux yeux de nombreux lecteurs, un contenu de référence en fonction duquel il est possible de déclarer que Dom Juan a tort et Sganarelle raison, c'est sans doute que ces lecteurs lisent (ou croient devoir lire) différemment un texte narratif et un texte dramatique: dans leur lecture, les versions du «récit» (au sens de F. Rastier) entrent différemment en concurrence selon qu'il s'agit d'établir la vérité d'un récit (cette fois au sens de Gérard Genette ${ }^{3}$ ) ou celle d'un drame. C'est donc à la question de la lecture du théâtre que nous sommes reconduits.

\section{La vérité du théâtre}

Tout indique dans l'affaire qui nous occupe que les discours des personnages ont un poids certain par rapport à la voix didascalique, qui semble privée de toute compétence en termes d'arbitrage. Cela donne provisoirement raison à la remarque d'Anne Ubersfeld: «il n’y a pas dans le texte de théâtre une voix privilégiée ${ }^{4}$ ». Dans son analyse du «modèle actantiel au théâtre», Ubersfeld note encore:

La présence des deux sujets, présence caractéristique du texte de théâtre, qu'elle soit le résultat d'une investigation textuelle ou d'une construction de l'objet «littéraire», met au jour l'ambiguïté fondamentale du texte de théâtre, cette ambiguïté que F. Rastier montre si bien à propos de Dom Juan, sans peut-être la rattacher assez clairement au statut théâtral du texte 5 .

1. Cela n'empêcherait d'ailleurs personne ni de s'identifier au personnage condamné, ni d'éprouver de la sympathie pour lui, ni même encore de trouver à son discours quelque sens ou pertinence.

2. Couton considère que les spectateurs, au début de l'acte $V$, sont également victimes de l'hypocrisie du libertin dans la mesure où, comme Dom Louis, ils sont susceptibles de croire à la sincérité de sa profession de foi (art. cité, p. 11).

3. «Je propose de nommer [...] récit [...] le signifiant, énoncé, discours ou texte narratif lui-même.» Voir Gérard Genette, «Discours du récit. Essai de méthode», Figures III, Paris, Seuil, 1972, p. 65-273, ici p. 72.

4. Anne Ubersfeld, Lire le théâtre I (1977), $2^{\mathrm{e}}$ éd., Paris, Belin, 1996, p. 79.

5. Ibid., p. 71. L'auteure souligne. 
On peut en réalité s'interroger sur la possibilité de faire passer au plan actantiel l'ambiguïté que F. Rastier conçoit sur le plan du « récit». Ce qui semble le permettre, en l'occurrence, est la non-lecture des énoncés didascaliques: ils sont la tache aveugle de toute analyse actantielle en même temps que celle de l'étude de F. Rastier qui, redisons-le, considère le Dom Juan de 1683, presque dépourvu de didascalies; les conclusions du sémioticien auraient sans doute été différentes s'il avait pris en compte les éditions de 1682 dans lesquelles le texte didascalique, on vient de le rappeler, arbitre le conflit entre le maître et son valet, réduisant sans doute de beaucoup l'ambiguïté du «récit», mais sans réduire l'ambiguïté en termes actantiels.

Il est toutefois important de noter avec Ubersfeld qu'en effet le "statut théâtral» de Dom Juan doit être pris en compte. Elle note qu'il est possible, sur le plan actantiel, que la représentation théâtrale "pose au spectateur le problème dramatique du texte $e^{1}$ " mais que "c'est la production de la représentation qui assure ou détruit l'ambivalence textuelle ${ }^{2} »$. Or cela est vrai aussi de l'ambivalence qui existerait sur le plan de la "vérité du récit». Sur scène, il est en effet possible que la vérité du récit soit la même que sur la page, mais il est aussi possible qu'elle diffère. Les énoncés didascaliques y sont généralement absents : le personnage, qui a la voix/voie libre, parle et présente une version du récit qui peut, parfois grâce au metteur en scène, parfois malgré lui, acquérir sur scène une valeur de vérité qu'elle n'avait pas sur la page. A l'inverse, la textualisation du spectacle est également en mesure d' "assure[r] ou [de] détrui[re] l'ambivalence» scénique lorsque celle-ci existe, c'est-à-dire d'agir sur la "vérité» - parfois volontairement sans doute, parfois aussi accidentellement. Réfléchissant au problème du mensonge au théâtre, Danielle Chaperon rappelle à propos de la mise en scène de textes préexistants:

[...] le metteur en scène peut faire des choix qui vont invalider les didascalies externes et même qui vont contredire les didascalies internes: il lui suffit de décider que le personnage, lorsqu'il les prononce, est ironique, mensonger ou dans l'erreur. C'est dire qu'un mensonge (dans le texte) peut devenir une vérité (sur la scène), simplement parce que cette vérité devient possible (et surtout intéressante ou troublante) dans les circonstances choisies par le metteur en scène. [...] De même une vérité (dans le texte) peut devenir (sur scène) une erreur manifeste ou une antiphrase. Un énoncé ironique (dans le texte) peut devenir (sur scène) un mensonge ou une assertion véridique. On conçoit que ces variations sont capitales et qu'elles peuvent modifier entièrement l'action telle qu'elle se présentait dans le texte dramatique. [...] En raison même de la dépendance cognitive des spectateurs envers les personnages, c'est le monde de la diégèse tout entier qui peut se métamorphoser. De la performance

1. Id. L'auteure souligne.

2. Ibid., p. 72. Je souligne. Elle commente ici une configuration qui ne correspond pas à Dom Juan, dans la mesure où la mise en scène de 1665 préexiste de longtemps aux éditions du texte, à moins de considérer le ou les manuscrits comme premiers, mais soit. 
dépend donc l'univers de la fiction lui-même, dans toutes ses dimensions: spatiales, temporelles et humaines ${ }^{1}$.

Plus généralement, il semble hasardeux de penser que le texte et le spectacle puissent toujours partager un même «récit» (au sens de F. Rastier). D. Chaperon est plus affirmative: "La fable, l'histoire racontée sur scène, malgré les fantasmes de fidélité qui ont pu être entretenus par certains metteurs en scène, ne peut pas être tout à fait la même que l'action suggérée par le texte ${ }^{2}$.» Quel que soit en fait le régime de cette (im)possible identité entre le "récit» sur la page et sur la scène, nous avons de toute façon intérêt, dans un premier temps, à distinguer Dom Juan comme texte et comme spectacle, ainsi que leur "vérité" respective.

Cette distinction permet de penser dans un second temps la manière dont Dom Juan comme texte est susceptible d'admettre plusieurs vérités. Car c'est bien de cela qu'il s'agit: la vérité du spectacle peut non seulement contredire celle du texte, mais se trouve encore en mesure de la contaminer. Si le texte dramatique est lu comme un texte de théâtre (c'est le présupposé d'Ubersfeld), la vérité de son «récit» se révèle en effet instable. En regard de la représentation théâtrale, il est notamment possible de considérer la voix didascalique, en mesure pourtant de remplir dans le texte une fonction métatextuelle, comme une voix en sourdine. Surtout aujourd'hui où «la pratique [...] de la mise en scène révèle que le texte secondaire [i.e. les didascalies] [...] n'assume pas une position de maîtrise et de surveillance par rapport au texte principal [i.e. le dialogue $]^{3}$ ", son silence scénique s'avère manifestement anticipé ou, dans le cas de Dom Juan, rétabli par de nombreux lecteurs du texte dramatique. Ce biais de lecture est habituel dans la critique française qui, par crainte de tomber dans les vieux réflexes de l'analyse de la «littérature dramatique», met peut-être un peu trop prestement un texte dramatique en relation directe avec des spectateurs. La volonté, légitime, de prise en compte de l'origine théâtrale ou du devenir théâtral du texte va en effet souvent de pair avec la non-considération, voire la non-lecture d'une partie ou de l'ensemble du texte didascalique, d'ailleurs exclu du texte dramatique par certaines des théories récentes du théâtre qui lui réservent plutôt un statut paratextuel. En chemin, on pourra tout simplement avoir oublié les lecteurs du texte analysé - opération qui nous renseigne sur la manière dont on pense devoir lire ou analyser le texte dramatique.

Ce qui est frappant en effet, c'est que Hallyn élabore son hypothèse en fonction d'un spectateur qu'il imagine: "Le spectateur ne peut-il pas se poser des questions?" Il justifie d'ailleurs la possibilité d'interpréter Dom Juan «sans y chercher [...]

1. Danielle Chaperon, «Le mensonge sur scène: la performance infinie», p. 120, dans Ambroise Barras, Eric Eigenmann (dir.), Textes en performance, Genève, MetisPresses, 2006, p. 107-124. L'auteure souligne. Sa réflexion prend appui sur la mise en scène par Didier Bezace au festival d'Avignon en 2001 d'une autre pièce de Molière: L'Ecole des femmes. Une version de cet article vient de paraître dans l'Atelier de Fabula (en ligne).

2. Id., «Ethique de la monstration scénique ou les "monstres" au théâtre», p. 143, dans Florence Quinche, Antonio Rodriguez (dir.), Quelle éthique pour la littérature? Pratiques et déontologies, Genève, Labor et Fides, 2007, p. 141-153.

3. Patrice Pavis, Dictionnaire du théâtre (1980), 2e édition (1996), Paris, Armand Colin, 2009, p. 357. 
l'expression d'une conviction religieuse», notamment par l'artificialité de la représentation du surnaturel sur la scène théâtrale : «En outre, la pièce impose elle-même, en tant que représentation, la suggestion d'une imitation artificielle d'une punition surnaturelle... ${ }^{1}$.» Pour Hallyn, tout se passe comme si la scène de théâtre elle-même - en général, ou celle de 1665 qu’il se représente en 1988? - invitait également à résoudre "les merveilles [par] les mathématiques ${ }^{2}$ ». D'une certaine manière, en extrapolant Hallyn, on pourrait dire - l'affirmation est paradoxale - que, si le texte didascalique est du côté de Sganarelle (et du Ciel), la représentation théâtrale est du côté de Dom Juan. Hallyn semble en effet voir dans le statut théâtral du texte un argument en faveur d'une lecture qui permet d'exclure le surnaturel de Dom Juan, comme si l'artificialité de la représentation du surnaturel sur scène induisait pour le lecteur du texte l'idée que la Statue et le Spectre sont artificiels dans l'univers de la fiction (et donc dans la représentation textuelle de celui-ci).

On ramènera donc l'ensemble de nos observations à trois constats: 1) La vérité du récit dans le texte dramatique peut être multiple (c'est-à-dire au moins double), selon que le lecteur convoque ou non un spectacle théâtral dans sa lecture, et surtout selon la façon dont il se représente ce spectacle. La vérité du récit écrit est dès lors tributaire d'une manière de lire le texte dramatique. 2) La vérité du texte dramatique lu comme un texte de théatre peut être contaminée dans la lecture non seulement par la vérité de la mise en scène qui en est historiquement à l'origine - elle se présenterait dans le texte écrit comme possiblement altérée -, mais aussi sans doute par la vérité de la mise en scène qui en est l'horizon possible - la vérité du récit ne serait alors dans le texte écrit que provisoire. Dès lors, dans tout texte dramatique lu comme un texte de théâtre, il n'existerait potentiellement plus aucun contenu de référence. On conçoit aisément que cette situation puisse avoir des conséquences proprement infernales pour l'analyse de texte ${ }^{3}$. 3) Le "récit" au théâtre peut transcender la frontière entre le textuel et le scénique. En vertu de ce phénomène, on préférera désigner autrement la "vérité du récit» d'un texte dramatique: on l'appellera plutôt la vérité du drame.

1. Hallyn, art. cité, p. 204 et 203. L'auteur souligne.

2. "Les "libertins" [...] attachent beaucoup d'importance à de tels "prodiges" [les automates actionnés par les hommes eux-mêmes sans qu'ils s'en aperçoivent], surtout pour insister sur l'explication purement rationnelle et combattre ainsi la superstition. [...] Les merveilles s'expliquent par les mathématiques. Faut-il rappeler à ce propos le credo de Dom Juan? "Je crois que deux et deux sont quatre, Sganarelle, et que quatre et quatre sont huit." (III, 1)» (Ibid., p. 202-203.)

3. ... sans compter qu'elle est déjà entravée par le caractère mobile de la perspective dramatique (non narrative) à l'œuvre dans ce type de textes. Sur la question du point de vue au théâtre, voir: Romain Bionda, "L'hésitation du spectateur. Le théâtre fantastique mode d'emploi (Hamlet)", dans Delphine Abrecht, Lise Michel, Coline Piot (dir.), Portraits de spectateurs de théâtre: faire ceuvre d'une réception, à paraître. 


\section{La vérité de Dom Juan}

On en vient ainsi à l'hypothèse que deux œuvres possiblement distinctes, les œuvres textuelle et scénique, portent ici le même titre - Dom Juan - et qu'elles forment conjointement une troisième œuvre, théâtrale, c'est-à-dire textuelle et scénique, qui les subsume: Dom Juan ${ }^{1}$. Dom Juan est donc le nom de trois ensembles pouvant être considérés chacun comme une œuvre (Dom Juan comme texte, comme spectacle et comme pièce de théâtre). Les éditions de 1682 ou de 1683 font-elles œuvre sans la mise en scène de 1665 (laissons de côté les mises en scène ultérieures par d'autres metteurs en scène) ou avec - et alors selon quelles modalités? Dans cette imbrication des œuvres résident la véritable difficulté et la source principale de l'ambiguïté propres à la lecture des textes dramatiques, qui n'ont pas le même statut selon qu' on les pense comme des œuvres à part entière ou du point de vue de l'auvre théâtrale, c'est-à-dire par exemple dans la perspective d'un théâtre considéré comme un «art à deux temps» (Henri Gouhier ${ }^{2}$ ) ou encore comme un "art allographique» (Nelson Goodman ${ }^{3}$ ). La conception que le lecteur peut avoir du degré d'autonomie opérale (totale, partielle ou nulle) du texte qu'il lit a sans doute un effet sur sa manière de le lire. Plusieurs facteurs entrent en jeu de façon complexe: notamment l'image que le lecteur se fait du théâtre contemporain à l'auteur du texte qu'il lit, mais aussi du théâtre contemporain à lui-même.

Selon Ubersfeld, "le texte est de l'ordre de l'indécidable; c'est la pratique qui constitue, construit le sens. Lire le théâtre, c'est préparer simplement les conditions de production de ce sens ${ }^{4} »$. Mais ce qui est de l'ordre de l'indécidable ici, ce n'est pas le sens du texte dramatique considéré en lui-même et pour lui-même: c'est d'une part le sens du texte considéré dans son rapport à une représentation théâtrale - de Dom Juan comme texte de théâtre -, d'autre part le sens de l'ensemble constitué par le texte et la représentation théâtrale - de Dom Juan comme pièce de théâtre. Si le texte dramatique est indécidable en lui-même, c'est au même titre que les textes narratifs (qui ne sont pas non plus de l'ordre du «décidable») : rappelons que les «trous » du texte dramatique chers à Ubersfeld (que le texte didascalique remplirait mal), Wolfgang Iser en voyait (légitimement) dans tous les textes.

Aujourd'hui au moins, la lecture du texte dramatique a cela de spécifique, par l'intermédiaire de ses plus grands enseignants, de demander à celui qui la pratique - qu'il soit critique professionnel ou non - de prendre en compte son éventuel «statut théâtral »: c'était le reproche qu'Ubersfeld adressait à F. Rastier à propos de sa "double lecture de Dom Juan». Jean de Guardia et Marie Parmentier observent de leur côté:

1. Les deux premières œuvres n'ont dès lors peut-être pas le même statut que la troisième.

2. Henri Gouhier, Le Théatre et les arts à deux temps, Paris, Flammarion, 1989. Voir aussi L'Euvre théatrale, Paris, Flammarion, 1958.

3. Nelson Goodman, Langages de l'art. Une approche de la théorie des symboles [1968, 1976], trad. Jacques Morizot, Paris, Arthème Fayard, 2011.

4. Anne Ubersfeld, Lire le théâtre I, op. cit., p. 226. 
Les circonstances matérielles des représentations passées, qui informent profondément les structures des textes de théâtre anciens, échappent très largement à la plupart des lecteurs, si bien que toute lecture scénique est, elle aussi, en un sens, de l'ordre de la fiction. La lecture scénique se veut plus adaptée à la nature de son objet que la lecture fictionnelle; pourtant, en adaptant à un texte des compétences [de spectateur] qui lui sont étrangères, elle crée bien souvent à son tour une scène fictionnelle ${ }^{1}$.

Or, il en va sans doute de même pour les historiens les mieux informés, dont le souci du caractère originairement théâtral d'un texte dramatique entraîne presque nécessairement sur la pente de la "fiction»-ou de l'imagination. En fait, cette imagination est mobilisée au sujet de toutes les mises en scène auxquelles le lecteur n'a pas assisté. C'est en partie pourquoi, même si elle s'applique prioritairement aux textes dont le lien avec le théâtre est avéré, cette manière de lire peut tout aussi bien s'appliquer à des textes dont le caractère théâtral n'est pas établi historiquement, mais seulement postulé par un lecteur. Comme le dit Véronique Lochert, le texte didascalique fait signe vers « un projet spectaculaire dont le degré de réalité est variable ${ }^{2}$ »: nul besoin qu'une représentation théâtrale ait bien eu lieu pour qu'un texte soit lisible comme texte de théâtre.

Trois remarques. 1) Cette imagination tend certes le plus souvent vers le passé du texte (par exemple la création de Dom Juan en 1665), mais elle peut aussi tendre vers son futur, qu'il soit antérieur au présent de la lecture (par exemple, peut-être, la mise en scène de J.-P. Vincent en 2012) ou même postérieur (futur simple d'une mise en scène "à faire»). 2) La "mise en scène» imaginée peut en outre interagir avec la «lecture fictionnelle», ou lecture diégétique: on l'a constaté dans la lecture de Hallyn, pour qui la nature de la représentation théâtrale de Dom Juan a des conséquences sur la nature (dès lors possiblement artificielle) du surnaturel dans l'univers de la fiction lui-même. 3) Serait donc spécifique à la lecture du texte dramatique pouvant être considéré comme théâtral non seulement la prise en compte d'une représentation théâtrale et sa capacité à interagir avec la lecture diégétique, mais encore la dimension temporelle de cette prise en compte: la lecture au passé, la lecture au futur (antérieur ou simple) et les interférences produites entre elles dans le présent de la lecture.

On peut en fait se demander quels liens elle entretient avec la tendance ordinaire de la lecture dite «littéraire» à la fabulation. Dans l'attention prêtée à ce que Richard Saint-Gelais distingue dans l'activité de lecture sous le nom de "parafictionnalisation ${ }^{3}$ " ("propositions [...] paraphrastiques, synthétiques ou conjecturales» à «orientation

1. Jean de Guardia, Marie Parmentier, «Les yeux du théâtre. Pour une théorie de la lecture du texte dramatique», p. 144, dans Poétique, n 158, 2009, p. 131-147. «Lecture scénique» ou "fictionnelle»: c'està-dire, dans le vocabulaire de J. de Guardia et M. Parmentier, qui tend vers la scène, ou vers la fiction.

2. Véronique Lochert, L'Ecriture du spectacle. Les didascalies dans le théâtre européen aux XVI et XVII siècles, Genève, Droz, 2009, p. 621.

3. "Convenons qu'une partie de la diégèse dépend de l'intervention du lecteur, qui développe silencieusement un discours hétérogène et discontinu: paraphrases à fonction d'élucidation, inférences, hypothèses, prévisions... Ce discours, je propose de le penser à l'aide de la notion de parafictionnalisation" (Richard Saint-Gelais, Fictions transfuges. La transfictionnalité et ses enjeux, Paris, Seuil, 2011, p. 457-458 pour nos premières citations). 
diégétique», c'est-à-dire "vers[ées] aussitôt au compte de la fiction » par le lecteur), le théoricien n'excepte pas le théâtre de son étude des relations qu'entretient la "transfictionnalité» avec le discours critique: Dom Juan est mentionné dès la deuxième ligne de Fictions transfuges. R. Saint-Gelais observe dans plusieurs commentaires d'une autre pièce de Molière:

De toute façon, le commentateur demeure prudemment vague en ne livrant qu'un fantôme d'épisode: manœuvre habile, qui demande au lecteur [du commentaire] d'admettre le principe d'un prolongement de la diégèse tout en reconnaissant qu'il ne revient pas au critique de s'avancer sur ce terrain risqué. Habile, aussi, en ce que cela suggère que le commentateur se tait faute de connaître le passé d'Arnolphe [au sujet notamment des maris qu'il aurait peut-être fait cocus], comme si l'obstacle tenait ici à l'ignorance - donc, à un facteur contingent - et non à un interdit statutaire (la clôture de la fiction) ou institutionnel (le partage des rôles entre écrivains et critiques) ${ }^{1}$.

R. Saint-Gelais conclut que la critique littéraire est "plus engagée qu'on ne le croit sur le terrain de la transfictionnalité», même si elle "ne propose [...], sages ou exubérantes, que des fictions réticentes ${ }^{2} »$. La traditionnelle double représentation de la fiction dramatique (textuelle et scénique) justifierait (et amplifierait) ainsi peut-être une tendance présente ailleurs, en invitant à des "prolongement[s] de la diégèse" susceptibles de "donne[r] un supplément d'existence à des personnages ${ }^{3}$ » - par l'intermédiaire notamment d'un spectateur imaginaire: "Ou bien... La fin de la pièce de Molière est extrêmement abrupte. Le spectateur ne peut-il pas se poser des questions ${ }^{4}$ ?"

L'hypothèse de Hallyn, qui avait pu sembler à première vue infondée en regard du texte de Dom Juan, s'appuie sinon se fonde en réalité sur le fonctionnement de la lecture des textes dramatiques telle qu'on la pense en France au sujet des textes que l'on considère "faits pour être représentés", selon la formule convenue. Cette lecture, parce que l'imagination y joue un grand rôle et de manière plutôt légitime - «il faut s'imaginer une mise en scène", entend-on souvent -, ouvre la voie à de telles hypothèses. Cela n'empêche pas qu'un lecteur puisse s'étrangler devant la proposition de Hallyn comme Armelle Héliot du Figaro devant «la trahison finale» de J.-P. Vincent à la Comédie-Française (s'il fallait le disculper, on pourrait alléguer un fait contingent: la scène du Théâtre Ephémère de la "Maison de Molière» ne disposant pas de trappe - la structure était provisoire -, Dom Juan ne pouvait pas facilement tomber dans l'abîme). Je préfère cependant retenir cette réflexion de Florence Naugrette sur le contresens de mise en scène:

[...] il est des écarts trop grands, trop douloureux, qui font souffrir le spectateur scandalisé par une torsion trop violente infligée [par le metteur en scène] au texte

1. Ibid., p. 487.

2. Ibid., p. 532.

3. Ibid., $4^{\mathrm{e}}$ de couverture.

4. Hallyn, art. cité, p. 204. Je souligne. 
sans défense, lui faisant dire ce qu'il ne peut dire, et le brisant, tandis que le spectateur, lui, refuse qu'on force sa souplesse jusqu'à la déchirure: ce grand écart-là, c'est le contresens de mise en scène ${ }^{1}$.

En effet, tout dépend du seuil de résistance du spectateur et de son sens de l'ouverture, ou simplement de son goût pour les «torsions» et les "grands écarts". Dans le cas qui nous occupe, on pourrait en dire autant du contresens de lecture: Hallyn, dans son hypothèse, fait en effet signe vers une mise en scène possible qui prend appui sur la «souplesse» du texte, et d'aucuns auront sans doute pu trouver qu’il la «force $[\ldots]$ jusqu’à la déchirure».

\section{La vérité du drame}

Dans l'article «Statue» du Dictionnaire de Don Juan, Sylvie Ballestra-Puech s'attache à son tour à «l'ambiguïté» de Dom Juan:

Molière maintient l'ambiguité jusqu'au dénouement [quant à la nature illusoire ou miraculeuse du mouvement de tête de la Statue], d'où la tentation récurrente, pour le metteur en scène comme pour le critique, d'exclure le surnaturel $[\ldots]^{2}$.

Le suspens éventuel est me semble-t-il peut-être moins dû à Molière qu'à une lecture «sceptique» qui profite d'une manière de lire les textes dramatiques. Le refus par les lecteurs d'accorder au texte didascalique une fonction métatextuelle et à ses énoncés une valeur de vérité est fréquent, pour plusieurs raisons: les didascalies sont perçues comme un "ajout" au "texte" dit sur la scène, comme un paratexte qu'on suppose parfois même hétérographique (cela pourrait être le cas des éditions de 1682 de Dom Juan: elles sont posthumes) et qu'on accuse de ne pas bien rendre compte de la dimension spectaculaire originelle dont le texte imprimé serait dans cette perspective une trace. Mais encore, implicitement, cette manière de lire le texte dramatique s'autorise peut-être aussi de la marge de manœuvre naturelle (et aujourd'hui légitime) de la mise en scène face aux indications scéniques contenues dans le texte.

Si la mise en scène réelle fonctionne en un sens comme laboratoire, voire comme preuve des lectures possibles des textes dramatiques, la "mise en scène» imaginée par le lecteur fonctionne comme embrayeur de la pluralisation non seulement du sens

1. Florence Naugrette, «Peut-on parler de contresens de mise en scène?», dans Intensités, «Le Contresens», $\mathrm{n}^{\circ}$ 2, présenté par Hélène Merlin-Kajman, hébergé sur le site Transitions, 2012. En ligne: http://www. mouvement-transitions.fr/index.php?option $=$ com_content $\&$ view $=$ article $\&$ id $=527$ : peut-on-parlerde-contresens-de-mise-en-scene \& catid = $45 \&$ Itemid = 196. Consulté le 24 février 2017.

2. Sylvie Ballestra-Puech, «Statue», p. 881-882, dans Pierre Brunel, Dictionnaire de Don Juan, Paris, Robert Laffont, 1999, p. 878-888. Larticle de Hallyn s’y trouve mentionné. 
des textes dramatiques, mais encore de leur «vérité». La valeur de vérité de la version du drame proposée par les didascalies est en effet affaiblie lorsqu'un texte dramatique est lu dans la perspective de sa (re)théátralisation avérée, supposée, postulée ou inventée, dans le passé ou le futur du texte, futur antérieur ou postérieur (c'est-àdire simple) au présent de la lecture. Le lecteur, parce que les textes dramatiques sont largement indécidables $d u$ point de vue du spectacle, est de ce même point de vue encouragé, voire autorisé à mettre en doute la "vérité» du texte - jusqu’à la mort de Dom Juan : ainsi, théâtralement, sa dernière réplique n'est-elle pas forcément sa réplique dernière. L'hypothèse de Hallyn, qui se nourrit de conjectures sur la représentation théâtrale, aura donc permis de montrer que la lecture des textes dramatiques pouvant être considérés comme théâtraux présente des spécificités (non naturelles, mais tributaires d'une histoire - théâtrale, éditoriale, théorique...) et des difficultés dont les conséquences sur l'exercice de l'analyse de texte (notamment en milieu académique, et plus généralement scolaire) mériteraient d'être pensées ${ }^{1}$.

Université de Lausanne

1. Merci à Danielle Chaperon et Marc Escola pour leur relecture sans terreur ni pitié. 